\title{
Vorwort zur 6. Auflage
}

Das „Berechnungsbuch" hat sich in dem Kreis der Instandsetzungsfachleute im Laufe der Jahre seines Erscheinens viele Freunde erworben. Wenn die Nachfrage in den letzten Jahren nicht prompt gedeckt werden konnte, so lag dies an den Nachkriegsschwierigkeiten, die am Verlagsort im besonders hohen Maße in Erscheinung traten und nur in mühseliger Kleinarbeit überwunden werden konnten.

Die jetzt vorliegende 6 . Auflage wurde überarbeitet und ergänzt. Infolge der Schwierigkeiten hinsichtlich der Drucklegung, die sich wesentlich länger als vorauszusehen war, hinzog, konnten jedoch nicht alle vorgesehenen Neuerungen in der Wickelei in der 6. Auflage berücksichtigt werden.

Es wird daher im Zusammenhang hiermit auf die laufenden Veröffentlichungen in der Fachzeitschrift „EMA“ - Die elektrische Maschine ${ }^{1}$ ) hingewiesen und insbesondere auf das in den Heften 1-2 1947 und 1-2 1948 bearbeitete Thema ,Verkürzte Wickelschritte bei Dreiphasenwicklungen" aufmerksam gemacht. Zum Zwecke der Überbrückung des in den Jahren 1945-1948 auf dem Fachbüchermarkt entstandenen Vakuums hat der Verfasser die Broschüre „Die Schnellbestimmung der Wickeldaten bei Drehstrommotoren, nach RaskopTabellen" herausgegeben, die ebenfalls als Ergänzung bei der Rekonstruktion verlorengegangener Wickeldaten zum Einsatz gelangen kann.

Krefeld, im Juni 1949

\section{Der Verfasser}

1) Technischer Verlag Herbert Cram, Berlin 30. 